\title{
Prácticas CEM enfocadas en la mitigación del Impulso Electromagnético del Rayo (IER): Earthing and Bonding; un enfoque de la IEC 62305
}

\author{
EMC Practices focused in the mitigation of \\ Lightning Electromagnetic Pulse (LEMP): Earthing \\ and bonding approach of the IEC 62305
}

\author{
Gabriel Malagón-Carvajal' \\ Wilson Giraldo-Picón ${ }^{2}$ \\ Gabriel Ordoñez-Plata ${ }^{3}$ \\ Julio Cesar Chacón ${ }^{4}$
}

Fecha de recepción: 5 de febrero del 2013

Fecha de aprobación: 14 de abril del 2013

I Ingeniero Electricista. Candidato a Máster en Ingeniería Eléctrica. Investigador Grupo de Investigación en Sistemas de Energía Eléctrica. Universidad Industrial de Santander. Carrera 27 con Calle 9. Colombia. Móvil: 3168290062. Correo electrónico: gabriel.malagon@correo.uis.edu.co

2 Ingeniero Electricista. Máster en Potencia Eléctrica. Investigador Grupo de Investigación en Sistemas de Energía Eléctrica. Profesor cátedra Titular de la Escuela de Ingenierías Eléctrica, Electrónica y de Telecomunicaciones - Universidad Industrial de Santander Carrera 27 con Calle 9. Colombia. Móvil: 300462730 I. Correo electrónico:wgiraldop@yahoo.com

3 Ingeniero Electricista. Doctor en Ingeniería Industrial. Investigador Grupo de Investigación en Sistemas de Energía Eléctrica. Profesor Titular de la Escuela de Ingenierías Eléctrica, Electrónica y de Telecomunicaciones Universidad Industrial de Santander Carrera 27 con Calle 9. Colombia. Móvil: 3002169526. Correo electrónico: gaby@uis.edu.co

4 Colombiano. Ingeniero Electricista. Máster en Potencia Eléctrica. Investigador Grupo de Investigación en Sistemas de Energía Eléctrica. Profesor asociado de la Escuela de Ingenierías Eléctrica, Electrónica y de Telecomunicaciones - Universidad Industrial de Santander - Carrera 27 con Calle 9. Colombia. Móvil: 31579|2529. Correo electrónico: cchacon@uis.edu.co 


\section{Palabras clave}

Compatibilidad electromagnética (CEM); Impulso electromagnético del rayo (IER); Sistema de puesta a tierra (earthing system); Red de equipotencialización (bonding network); Sistema de terminales de puesta a tierra (earth-termination system); perturbaciones electromagnéticas.

\section{Resumen}

Este artículo presenta los resultados obtenidos durante la identificación de parámetros de compatibilidad electromagnética en sistemas de protección contra rayos. En él se exponen una serie de buenas prácticas de ingeniería orientadas a la mitigación de las perturbaciones de tipo transitorio, creadas por el impulso electromagnético generado durante la descarga a tierra de un rayo. Estas perturbaciones se ven a menudo reflejadas como sobretensiones y/o sobrecorrientes radiadas o conducidas, también llamadas surges, las cuales inciden fuertemente sobre la operación de las instalaciones eléctricas de uso final, trayendo consigo costos significativos por paradas de los procesos asociados a la instalación y los equipos dentro de esta. Las recomendaciones aquí planteadas se centran en la temática relacionada con los sistemas de puesta a tierra descritos en la norma IEC 62305-4, 20 I0, en la cual se describen la red de equipotencialización (bonding network) y el sistema de terminales de tierra (earth-terminal system) como componentes básicos del sistema de puesta a tierra (earthing). Estas prácticas o recomendaciones se orientan a su puesta en marcha durante la etapa de diseño de la instalación, vinculando así las especialidades de arquitectura, ingeniería civil e ingeniería eléctrica, puesto que en su mayoría deben ser implementadas durante la construcción de la instalación. Finalmente, en este documento se describen las configuraciones básicas de la red de equipotencialización, revisando sus principales características, ventajas y componentes principales; a la vez, se abordan las principales configuraciones del sistema de terminales de tierra y sus ventajas frente a la mitigación de las perturbaciones electromagnéticas.

\section{Keywords}

Electromagnetic compatibility (EMC); LEMP (Lightning Electromagnetic Compability); earthing system; bonding network; earth-termination system; Electromagnetic disturbances.

\section{Abstract}

This paper presents the results obtained during the parameter identification of electromagnetic compatibility in lightning protection systems. It sets out a number of good engineering practices, aimed at mitigation of disturbances, transient type, created by an electromagnetic pulse, generated during the grounding of lightning. These disturbances are often reflected as overvoltage and / or overcurrent radiated and conducted or also called surges, the latter a strong impact on the operation of end use electrical installations by bringing significant cost stops the processes associated with the installation and equipment within the same. The recommendations raised here focus on the issues related to grounding systems described in the standard (IEC 62305-4, 2010), which describes the equipotential network (bonding network) and system ground terminals (earth-terminal system) as basic components of the system grounding (earthing). These practices or recommendations are aimed at start-up, during the design stage of the system, thereby linking the specialties of architecture, civil engineering and electrical engineering, as most should be implemented during the next stage of design, as is the construction of the installation. Finally, this paper describes the basic configurations of equipotential network reviewing the main features, advantages and main components, in turn addresses key system configurations ground terminals and its advantages over electromagnetic interference mitigation. 


\section{Introducción}

Las descargas atmosféricas son eventos naturales de muy alta energía, que ponen en riesgo la seguridad de los seres vivos y el adecuado funcionamiento de los sistemas eléctricos y electrónicos o cargas sensibles. Así mismo, el aumento progresivo de las cargas no lineales ha hecho que las directrices para el diseño usadas desde hace algunos años con respecto al sistema de puesta a tierra y el sistema de protección contra rayos, no sean suficientes.

Partiendo del origen de la problemática por incompatibilidad electromagnética al interior de las instalaciones eléctricas y tomando como principales causas: el Impulso Electromagnético del Rayo (IER'), generado a partir de las descargas atmosféricas, la calidad de la onda del sistema de suministro y los mismos equipos como fuentes de perturbaciones, es evidente que se deben identificar alternativas de mitigación que deberán aplicarse desde el diseño para garantizar una adecuada construcción y operación de la instalación de uso final.

Al hacer una comparación entre los cientos de megajoules de energía que se generan a través del IER con sus diversos fenómenos relacionados y los milijoules que pueden soportar los sistemas sensibles, es claro que se deben tomar medidas adicionales que conlleven la protección de estos equipos y de la instalación y la disminución del riesgo eléctrico. En este sentido, un diseño en el que se toman medidas de protección, orientadas principalmente a la reducción del riesgo de daño físico en estructuras y la seguridad de los seres vivos, pero en el que no se consideren los problemas inherentes a la calidad de la energía y la incompatibilidad electromagnética de sistemas eléctricos y electrónicos, puede traer consigo gastos adicionales, que impactarán en las etapas de operación y mantenimiento (Chapman, 200I).
La necesidad de identificar soluciones oportunas a la problemática expuesta se da a partir de los crecientes costos que este tipo de fallas puede originar en los sistemas eléctricos y electrónicos, debido a la salida de operación de equipos y a la importancia del proceso asociado a su funcionamiento (Baggini \& Bua, 2004). Es por esto que se busca reconocer buenas prácticas a través de la investigación en estándares y normas internacionales, así como artículos técnicos relacionados con esta temática. En la estructura de este artículo, se presenta en la sección II las generalidades sobre la mitigación de influencias electromagnéticas definiendo el entorno electromagnético, los mecanismos de mitigación o control y las fuentes de interferencia o perturbaciones. En la sección III se abordan aquellas prácticas de ingeniería orientadas a la mitigación de los fenómenos ocasionados por la incompatibilidad electromagnética debidos al IER, resaltando las ventajas y características de un sistema de puesta a tierra que aborde la red de equipotencialización y el sistema de terminales de puesta a tierra como componentes básicos de un único sistema completo de puesta a tierra. Por último, se presentan algunas conclusiones obtenidas durante el desarrollo de la presente investigación.

\section{Generalidades sobre la mitigación de influencias CEM}

\section{(Overview of mitigation EMC influences)}

\section{Control básico de la interferencia} (Basic Control of Interference)

En su forma más simple, el problema de la interferencia electromagnética esta compuesto por una fuente de perturbación, una víctima y un medio entre los dos (ver figura I). El control de la

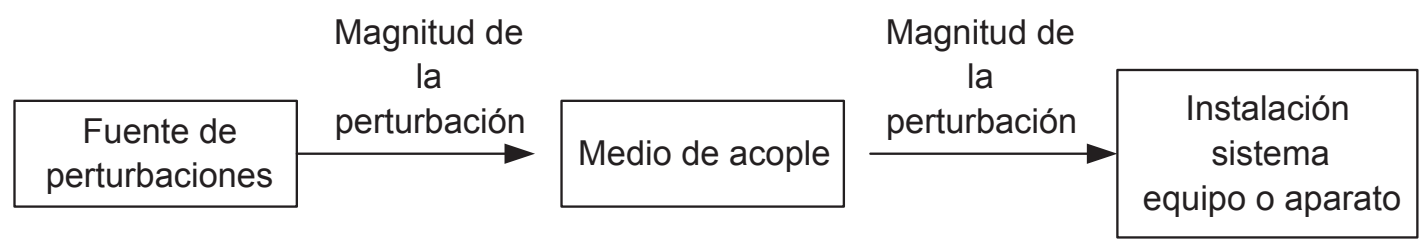

Figura I. Esquema básico de CEM.

Fuente: Langguth, 2004. 
interferencia consiste en suprimir la fuente de interferencia y el fortalecimiento de la víctima, o impedir la interacción fuente-víctima a través del medio.

Dependiendo de la naturaleza de cada uno de los elementos anteriormente nombrados, se pueden establecer prácticas que permitan reducir los efectos de las perturbaciones que generan incompatibilidad electromagnética dentro y fuera de la instalación. Cuando se orienta el control hacia la fuente, como en el caso de un rayo, y ante la imposibilidad alguna de ello, el control de la interferencia debe ser relegado al medio, el cual se realiza básicamente mediante la definición de zonas de protección, el dimensionamiento de blindajes e interfaces de aislamiento y un buen diseño de un sistema de puesta a tierra, entre otros. Por otra parte cuando el criterio para el control de la interferencia se orienta a la protección de las víctimas, la estrategia de mitigación se orienta a la implementación de la práctica, lo más cerca de las victimas del IER, las cuales en este caso son las cargas sensibles.

Fuentes de perturbaciones

(Sources of Interference)

Las fuentes de incompatibilidades electromagnéticas se clasifican de acuerdo con diferentes parámetros y características. Las principales categorías se dan según los siguientes parámetros (Schneider Electric, 2004):

\section{El origen}

Las de origen natural incluyen el ruido atmosférico terrestre debido a tormentas, descargas electroestáticas, emisiones de rayos procedentes del sol y del cosmos que inciden sobre el planeta. Las de origen humano incluyen tanto las radiaciones intencionadas, ligadas fundamentalmente a los equipos de comunicaciones, como las no intencionadas, como, por ejemplo, los armónicos provocados por el funcionamiento de cargas no lineales.

\section{El espectro en frecuencia}

En esta categoría se encuentran las de banda estrecha, que son fuentes de señal con frecuencias discretas tales como: transmisores y receptores de radio, radares, generadores industriales de alta frecuencia, hornos de microondas, equipos de soldadura, receptores de sonido, dispositivos de ultrasonido y convertidores de corriente. También se encuentran las de banda ancha, que son potentes disruptores en las instalaciones electrónicas de automatización debido a las altas frecuencias asociadas a su funcionamiento. Entre los principales generadores de este tipo de perturbaciones tenemos: motores, lámpara de descarga, circuitos de control con semiconductores, dispositivos de conmutación, descargas electrostáticas, efecto corona y explosiones nucleares.

\section{La frecuencia con la que se presenta la perturbación y el tiempo que tarda en desaparecer}

Existen perturbaciones en las que la influencia sobre la instalación es momentánea o transitoria. Los efectos aparecen en la instalación y en poco tiempo desaparecen, pero aun cuando la duración es corta las consecuencias pueden llegar a ser graves, como en el caso de las descargas atmosféricas a tierra. También hay perturbaciones en las que la influencia sobre la red es constante y la afectación en la instalación se puede dar por sobrexposición de los elementos de esta a dichas perturbaciones, como en el caso de las distorsiones armónicas que causan calentamiento excesivo sobre conductores y dispositivos de protección, provocando pérdidas eléctricas y disparo de las protecciones.

\section{Buenas prácticas orientadas a la mitigación} de perturbaciones cem ocasionadas por el IER (good practices aimed at mitigation of disturbances EMC caused by lemp ${ }^{2}$ )

Se entiende por buenas prácticas el conjunto de acciones que han rendido un buen servicio, o incluso excelente, en un determinado contexto y que se espera que en contextos parecidos rindan similares resultados.

A continuación se relaciona una serie de buenas prácticas acogidas por la Comisión Electrotécnica Internacional (IEC) a través de sus normas IEC 62305-4, 2010 e IECTR 61000-5-6, 2002, junto con algunas otras presentadas por fabricantes de dispositivos de protección. 
Sistema de puesta a tierra y equipotencialización (Earthing system and Bonding)

El sistema de puesta a tierra (SPT) forma parte del sistema de protección externa y es el encargado de conducir y dispersar las corrientes del rayo en el suelo. Comprende toda unión directa de los equipos eléctricos y electrónicos con la tierra o una masa metálica, con el objeto de conseguir que el conjunto de instalaciones, construcciones y superficies próximas al suelo no estén sometidas a diferencias de potencial peligrosas (tensiones de paso y de contacto) y que al mismo tiempo permita el paso a tierra de las corrientes de falla o de rayo.

Para ello, algunos elementos de un $\mathrm{SPT}^{3}$ pueden disponerse para que cumplan una función específica dentro de cada instalación de uso final, pero cada uno de estos forma parte de un único sistema. Es de esta forma como la norma IEC 623054, 2010 propone que todos los elementos de un SPT estén conectados entre sí, formando lo que se conoce como una red de equipotencialización $\left(\mathrm{RE}^{4}\right)$. Esta red de equipotencialización y el sistema de terminales de puesta a tierra (TPT) forman un sistema completo de puesta a tierra (SPT), con el cual se aseguran los siguientes parámetros:

I. La protección de las personas, limitando las tensiones de paso y de contacto a valores seguros.

2. La protección de instalaciones contra daños por rayos.

3. La compatibilidad electromagnética (CEM) para la mitigación de perturbaciones electromagnéticas.

4. El correcto funcionamiento del sistema de distribución, asegurando la calidad en el suministro de energía eléctrica.

Por su parte, la red de equipotencialización tiene como función interconectar el sistema de terminales de puesta a tierra ${ }^{5}$ con todas las partes conductoras de la estructura y del sistema interno (sistemas eléctricos y electrónicos dentro de una estructura, excluyendo los conductores activos), con el fin de reducir las diferencias de potencial (tensiones de paso y de contacto) y el campo magnético causado por las corrientes del rayo. El sistema de terminales de puesta a tierra puede estar compuesto por:

I. Electrodos: Parte o grupo de partes del sistema de terminales de puesta a tierra que suministra contacto directo eléctrico con la tierra y dispersa las corrientes del rayo en ésta. Pueden ser verticales, horizontales o se puede utilizar la cimentación de acero reforzado de la estructura. En este último caso se conocen como electrodos de puesta a tierra de cimentación.

2. Anillos: Electrodos a tierra que forman un lazo cerrado alrededor de la estructura. Interconectan las bajantes para distribuir las corrientes de rayo entre ellos.

3. Enmallados: Interconexión de los terminales de sistemas de puesta a tierra de varias estructuras.

En la figura 2 se presenta un ejemplo de un sistema de puesta a tierra completo en el que se ha construido una red de equipotencialización a lo largo, alto y ancho de la estructura, junto con la posterior equipotencialización del sistema de terminales de puesta a tierra. Este último puede estar constituido por electrodos naturales como: el acero estructural de la edificación, las tuberías metálicas de los servicios entrantes (agua, gas, energía y telecomunicaciones) y los electrodos formados a partir del acero figurado de placas prefabricadas y fundidas para la cimentación de la edificación (electrodos de puesta a tierra de la cimentación).

Como buena práctica, se recomienda que el electrodo de tierra del anillo equipotencial alrededor de la estructura y el electrodo de tierra del anillo equipotencial ${ }^{6}$ dentro de la estructura estén unidos a los electrodos de puesta a tierra de la cimentación, es decir, que estos componentes deben formar una red enmallada dentro y alrededor de la estructura teniendo un ancho de la malla generalmente de $5 \mathrm{~m} \times 5 \mathrm{~m}$ en el interior. Para estructuras adyacentes o contiguas no separadas longitudinalmente más de 30 metros, se recomienda un ancho de malla de $20 \mathrm{~m} \times 20 \mathrm{~m}$. Por encima de

3 Sistema de Puesta a Tierra.

4 Red de Equipotencialización. Bonding Network en idioma inglés.

5 Conjunto de elementos conductores que proveen un contacto eléctrico directo con el suelo y dispersan las corrientes de rayo a tierra.

6 Ring Earth Electrode, en idioma inglés. 


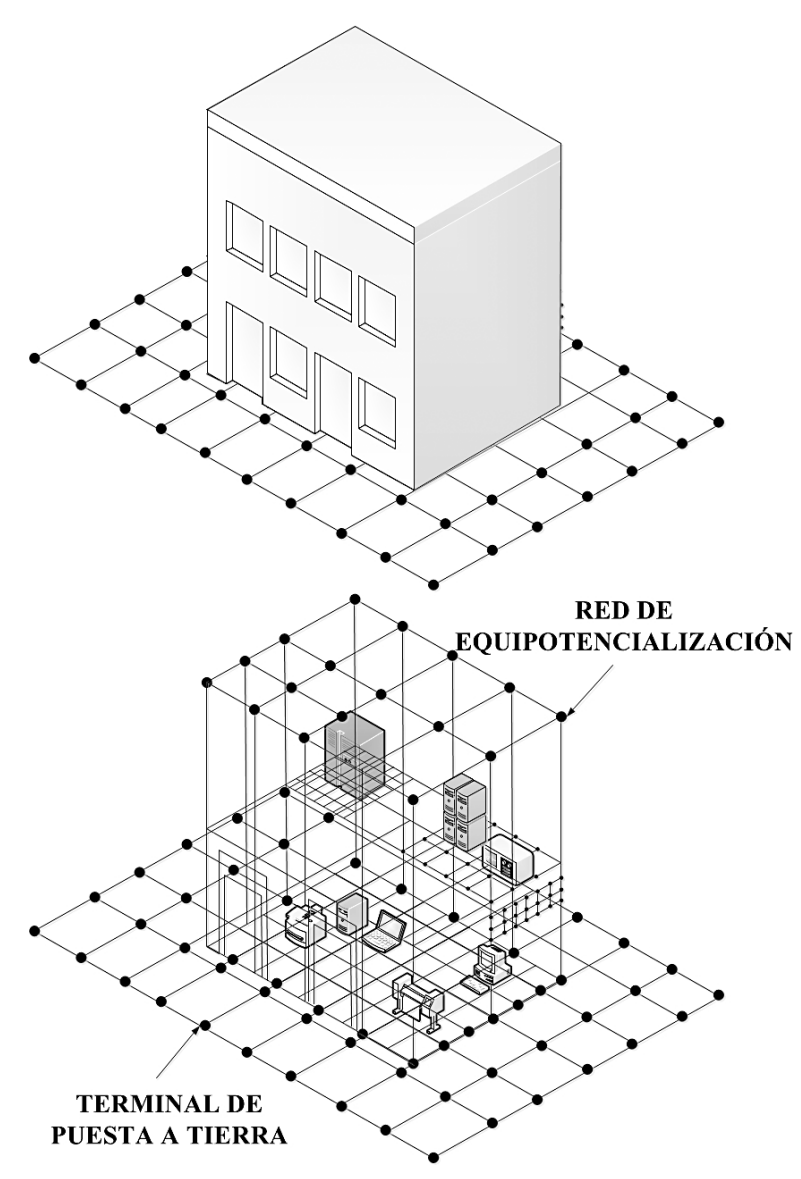

Figura 2. Red de equipotencialización y terminal de puesta a tierra.

Fuente: IEC 62305-4, 2010.

esta distancia de separación, las mallas pueden ser ensanchadas a $40 \mathrm{~m} \times 40 \mathrm{~m}$.

La premisa fundamental para las configuraciones de malla es una mínima impedancia del sistema de puesta a tierra y la equipotencialización de las partes metálicas no energizadas a través de la red de equipotencialización, permitiendo de esta forma una optimización del diseño de un Sistema de Protección contrarrayos, puesto que el sistema soportará una mayor disipación de las corrientes presentes ante una descarga atmosférica y una disminución de las diferencias de potencial peligrosas.

En la figura 3 se presenta una red de interconexión para un sistema de puesta a tierra de una instalación industrial, en la que se puede apreciar una tarea bastante difícil, si no imposible, pues no es tan sencillo garantizar la conexión a tierra de los bajantes y el uso apropiado de la cimentación, crucial para lograr

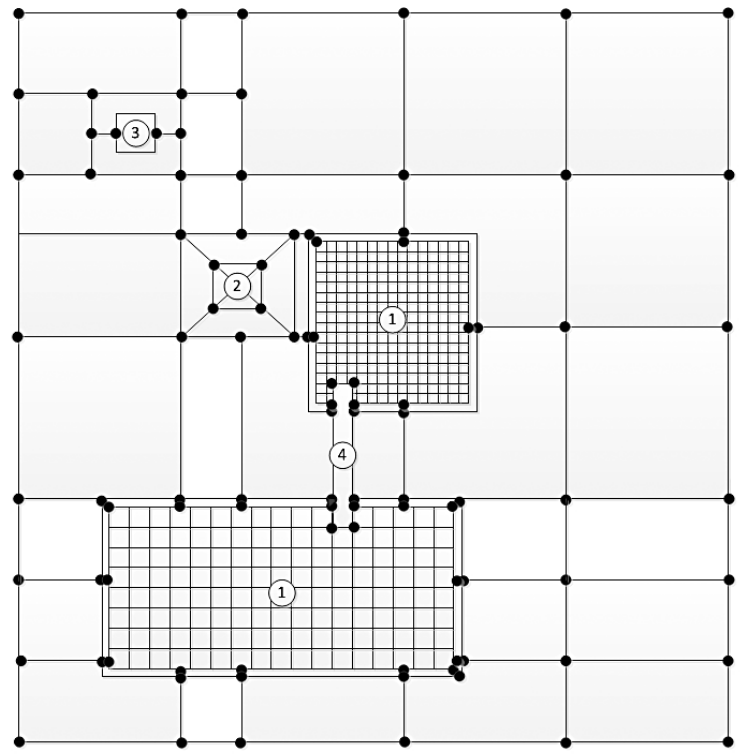

1. Construcción de una red mallada con los electrodos de puesta a tierra de la cimentación.

2. Torre de comunicaciones al interior de la planta.

3. Equipos en partes externas a las edificaciones.

4. Zanjas portacables.

Figura 3. Terminal del sistema de puesta a tierra en forma de malla.

Fuente: IEC 62305-4, 2010.

un efectivo sistema de puesta a tierra (mínima impedancia y la equipotencialización) en una edificación.

Por ello, se recomienda que desde las primeras etapas del diseño se considere la resistividad del suelo y la naturaleza del terreno donde se va construir la edificación. Esta información es importante y puede condicionar el diseño de la construcción. Por esta razón, es primordial realizar reuniones previas al inicio de la construcción entre los diseñadores del sistema de puesta a tierra, arquitectos y constructores con el fin de lograr un buen resultado con alta seguridad al mínimo costo.

\section{Red de equipotencialización \\ (RE) (Bonding Network)}

La RE es el conjunto de conductores que conectan las partes metálicas no energizadas del sistema eléctrico y la estructura de una instalación con el terminal de puesta a tierra, permitiendo disminuir las diferencias de potencial peligrosas ante la circulación de una corriente de falla y su respectiva disipación a tierra. 
Es necesario que la RE tenga una baja impedancia para evitar diferencias de potencial peligrosas entre todos los equipos en el interior de las ZPR ${ }^{7}$. Esto se puede lograr equipotencializando las partes conductoras de la estructura con las partes conductoras del sistema interno, elementos metálicos y servicios entrantes en material conductor, directamente en la frontera de cada ZPR o mediante el adecuado uso de dispositivos de protección contra surges ${ }^{8}$ (DPS), trabajando junto con la red de equipotencialización, la cual reduce el campo magnético de fuentes externas (ver IEC 62305-4, 20 I0, Anexo A).

Cuando se establecen uniones equipotenciales en los sistemas internos, las corrientes parciales del rayo (provocadas por el campo magnético de éste) pueden fluir dentro de estos sistemas. Por lo tanto, este efecto debe ser considerado en el diseño de las zonas de protección y la coordinación de DPS?. Estas uniones equipotenciales a menudo son provistas por conductores para aparatos dentro de una misma zona de protección, y a través de un DPS cuando la unión equipotencial que se busca está dada entre diferentes zonas de protección.

La $R E^{10}$ se puede diseñar como una estructura de malla tridimensional, con un ancho de malla típico de $5 \mathrm{~m}$ (ver figura 3). Esto requiere múltiples interconexiones de componentes metálicos sobre la estructura (tales como los refuerzos de acero en concreto, rieles del ascensor, grúas, cubiertas metálicas, fachadas metálicas, marcos metálicos de ventanas y puertas, marcos metálicos del piso, tuberías de servicio y bandejas portacables), junto con las barras de equipotencialización y los blindajes magnéticos de la ZPR. En la figura $4 a$ y la figura 5 se muestra un corte
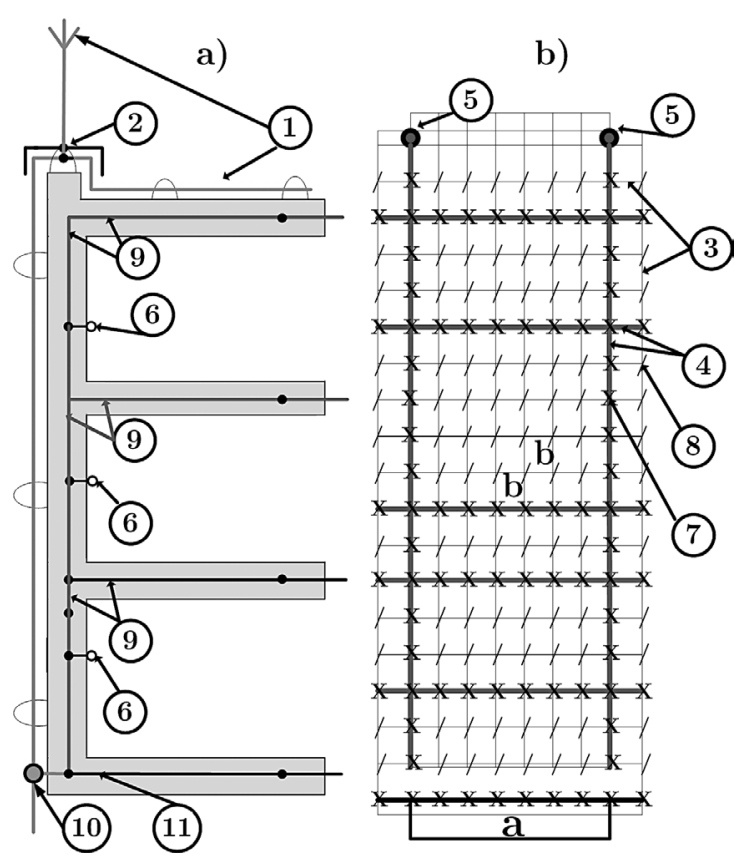

Donde:

I. Bajantes y sistema de captación del rayo (línea azul).

2. Cubierta metálica para el soporte del techo.

3. Acero de refuerzo de la estructura.

4. Conductores de malla superpuestos sobre el acero de refuerzo.

5. Unión común de conductores de malla.

6. Unión común para una barra de equipotencialización interna.

7. Conexión hecha mediante sujeción o soldadura, en la que se debe garantizar una unión firme de los conductores de malla y acero de refuerzo de la estructura.

8. Conexión arbitraria. Una conexión propia del acero de refuerzo de la estructura, comúnmente se hacen a través de sujeción hecha por alambres (empalmes).

9. Acero reforzado en concreto (con los conductores de malla sobrepuestos).

10. Anillo del electrodo de puesta a tierra (punto verde). Es aquel en el cual se equipotencializan todos los electrodos de un sistema de puesta a tierra.

1।. Electrodos en cimentación. Es aquel acero de refuerzo que está en contacto con la tierra.

a: Distancia típica de $5 \mathrm{~m}$ para los conductores superpuestos en la malla.

b: Distancia típica de I m para la conexión de esta malla con los refuerzos.

Figura 4. Utilización de las varillas de refuerzo de una estructura para la unión equipotencial.

Fuente: IEC 62305-4, 2010.

$7 \quad$ Zona de protección contra rayos; Lightning Protection Zone en idioma inglés.

8 Surges en idioma inglés está definido como Transitorio creado por un impulso electromagnético que aparece como una sobretensión y/o sobrecorriente.

9 Dispositivo de protección contra surges, SPD en idioma inglés.

I0 Red de equipotencialización, Bonding Network en idioma inglés. 


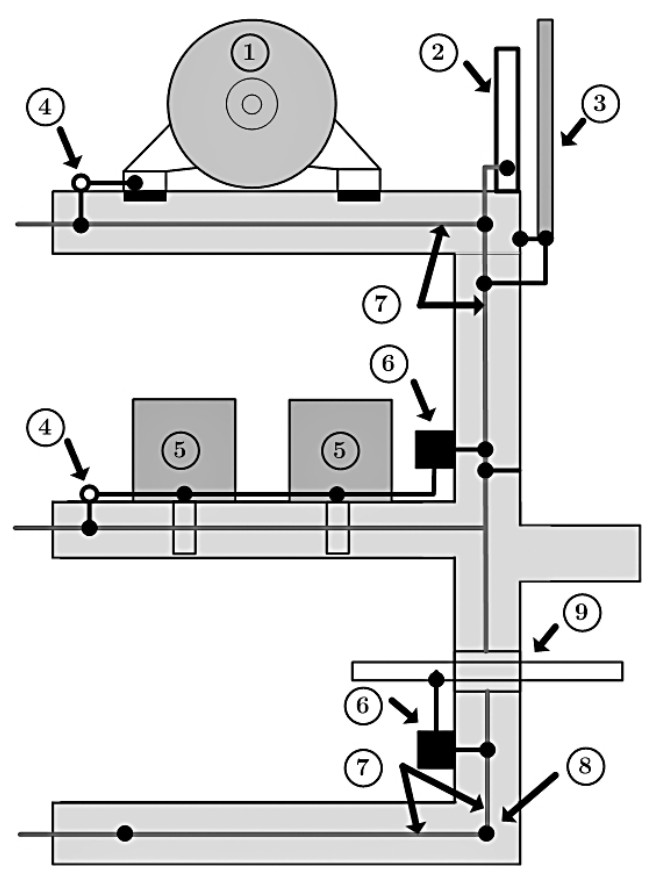

I. Equipo eléctrico (motor, generador)

2. Vigas de acero

3. Cubierta metálica de la fachada

4. Conjunto de interconexiones

5. Equipos eléctricos o electrónicos

6. Barra de equipotencialización

7. Acero de refuerzo en concreto (con los conductores de malla sobrepuestos)

8. Cimentación de los electrodos del SPT

9. Punto común para los diferentes servicios entrantes

Figura 5. Unión equipotencial en una estructura con refuerzos de acero.

Fuente: IEC 62305-4, 2010.

transversal de una edificación. En la figura 4b se observa una vista de planta, en la que se muestra la forma en que se debe integrar el acero de refuerzo con la RE.

\section{Configuración de la red de equipotencialización (Bonding Network Configuration)}

Las partes conductoras (gabinetes, recintos" estantes) y el conductor de protección (PE) de los sistemas internos (sistemas eléctrico o electrónico) deben conectarse a tierra mediante una red de equipotencialización, de acuerdo con las configuraciones presentadas en la figura 6 y descritas en los literales derivados de este ítem.

Cl. Configuración en estrella (S): Configuración en la cual todos los componentes metálicos de los sistemas eléctricos y electrónicos deben ser aislados adecuadamente del SPT. Cuando se utiliza la configuración S, todas las líneas energizadas de cada uno de los equipos deben ser cableadas, de tal forma que dichos conductores queden en paralelo y cercanos a los conductores de equipotencialización o PE (Protective Earth Conductor), siguiendo la configuración en S, con el fin de evitar bucles de inducción, dado en que a mayor área de inducción (tamaño del bucle) se puede presentar una mayor sobretensión ante la incidencia de un campo magnético.

La configuración S se puede utilizar cuando los sistemas internos estén ubicados en zonas relativamente pequeñas, donde las líneas que entran en la zona lo hacen por un solo punto.

C2. Configuración en estrella (SS): Configuración en donde la configuración S básica se integra al sistema de puesta a tierra mediante una barra de equipotencialización, que actúa como punto de referencia de tierra (PRT).

C3. Configuración enmallada (M): Configuración básica en donde todos los componentes metálicos de los sistemas eléctricos y electrónicos, también llamados sistemas internos (por ejemplo, gabinetes, recintos, estantes), no deben ser aislados del SPT. La configuración $M$ es preferida para sistemas eléctricos y electrónicos extendidos sobre zonas relativamente amplias, o en estructuras donde muchos de 


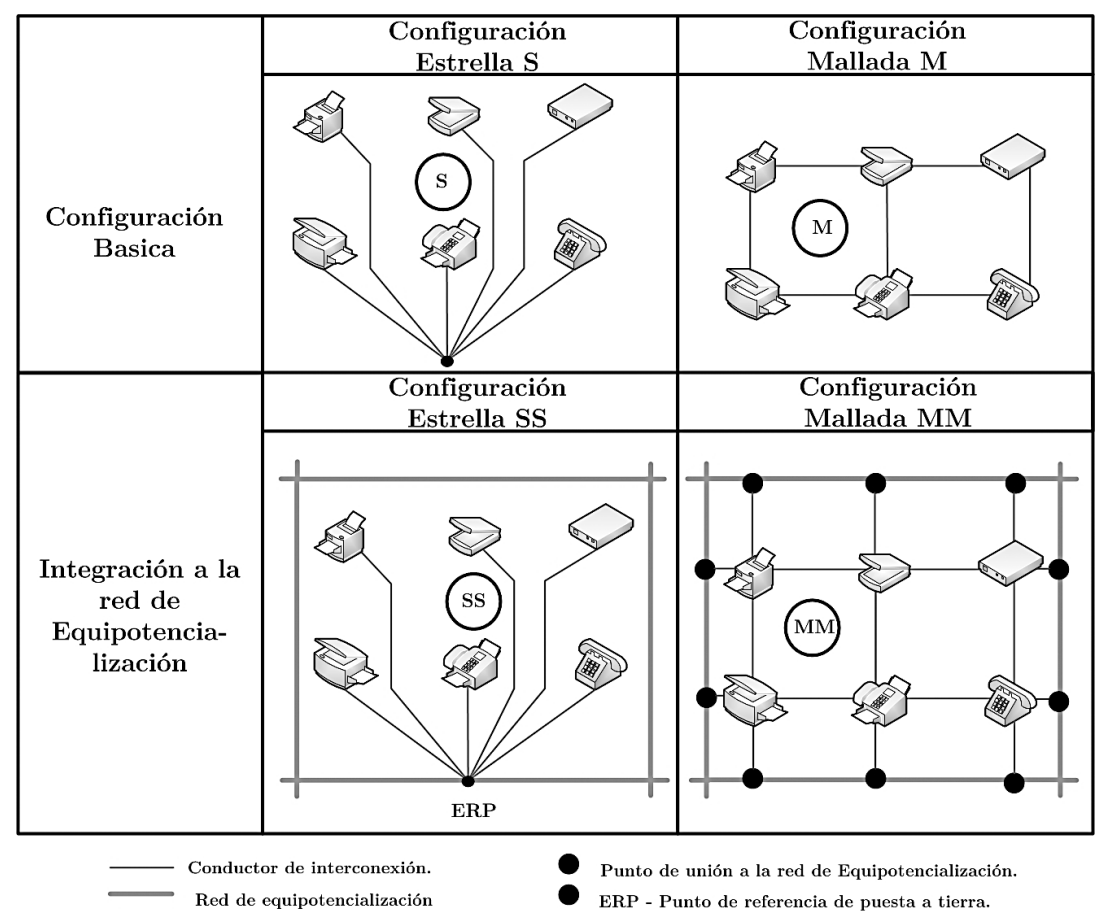

Figura 6. Integración de las partes conductoras de los sistemas internos a la red de equipotencialización. Fuente: IEC 62305-4, 2010.

los conductores se encuentran entre las partes individuales de los equipos y en donde las líneas entran a la estructura por diferentes puntos.

C4. Configuración enmallada (MM): Configuración en donde la configuración enmallada básica se integra al sistema de puesta a tierra mediante múltiples puntos de equipotencialización (Bonding Points), a la Red de equipotencialización. En sistemas complejos, las ventajas de las configuraciones $S$ y $M$ pueden ser combinadas como se muestra en la figura 7. Como resultado, se obtiene una combinación I (SS combinada con MM) o una combinación 2 (MS combinada con MM). revisar lo amarillo, parece faltar algo, un verbo quizás.

\section{Sistema de Terminales de Puesta a \\ Tierra (Terminal Earthing system)}

Este sistema tiene como función dispersar y disipar la corriente del rayo en la tierra, que es conducida por los bajantes después de la descarga atmosférica. En un sistema de protección externo se busca un bajo valor de resistencia de puesta a tierra (del orden de los $10 \Omega$, frente a transitorios de baja frecuencia). Así mismo, es recomendable que estos estén integrados con todos los demás sistemas de puesta a tierra por medio de uniones que garanticen la equipotencialidad en todas las condiciones de operación. En general, la norma IEC 62305-3, 2010 considera dos tipos básicos de configuraciones de terminales de puesta a tierra, conocidas como la de tipo A y la de tipo B, las cuales se describen a continuación:

DI.Configuración tipo A: Este tipo de configuración se compone de un terminal de puesta a tierra que incluye dos 0 más electrodos horizontales ${ }^{12}$ o verticales, instalados fuera de la estructura a ser protegida y conectados a cada uno de los bajantes del sistema de protección externa. Esta configuración se utiliza para estructuras bajas, en sistemas de protección con varillas y cables extendidos o para un sistema aislado. De acuerdo con la referencia IEC 62305-3, 2010, el sistema de terminales de puesta a tierra tipo $A$ debe instalarse a una profundidad de al menos $0,5 \mathrm{~m}$ en su parte superior, distribuidos 


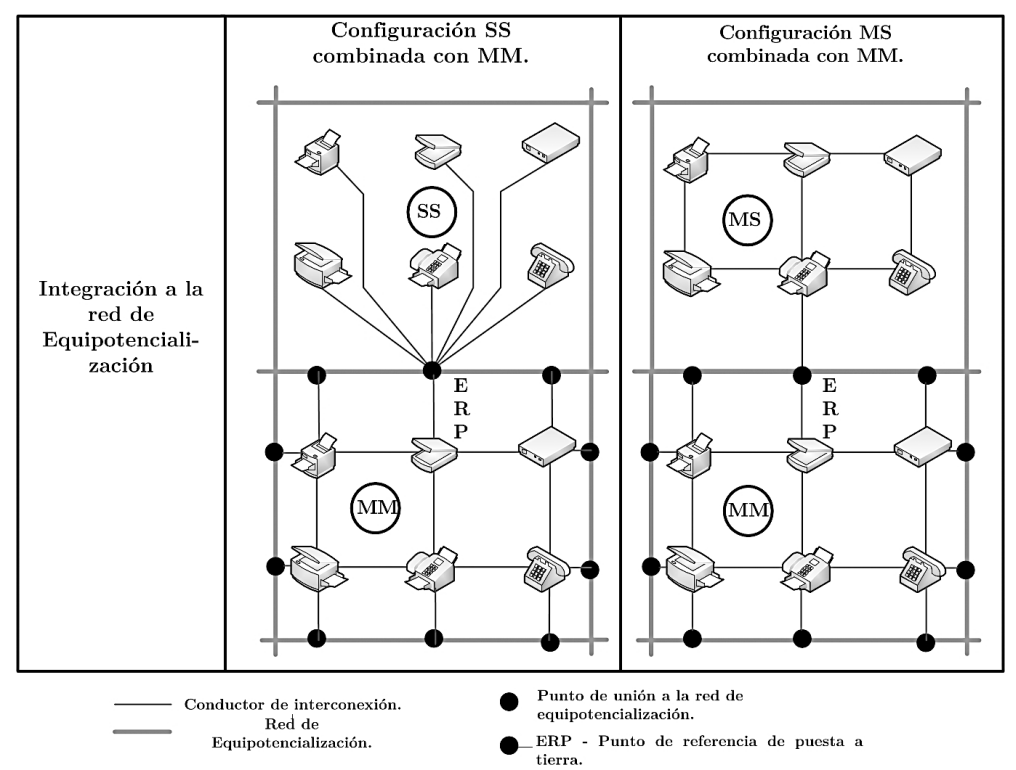

Figura 7. Combinaciones de los métodos de integración de las partes conductoras de los sistemas internos a la red de equipotencialización.

Fuente: IEC 62305-4, 2010.

uniformemente para poder minimizar efectos de acople eléctrico en la tierra y de tal forma que permita su inspección durante la construcción.

Por otra parte, en la figura 8 se muestra un esquema general de la configuración tipo A en una edificación.

D2. Configuración tipo B: Esta configuración está conformada por un terminal de puesta a tierra que incluye un anillo conductor externo ${ }^{13}$ a la estructura a proteger y el cual debe estar en contacto con el suelo en por lo menos un $80 \%$ de su longitud total o en contacto con los electrodos de la cimentación - electrodos a tierra, los cuales a su vez deben estar interconectados. Estos electrodos también pueden ser del tipo mallado.

En esta configuración, el número de electrodos no debe ser menor que el número de bajantes, con un mínimo de dos. Los electrodos adicionales deben ser conectados al anillo de puesta a tierra en los puntos en donde las bajantes son conectados a éste y en lo posible deben ser equidistantes. Así mismo, el anillo perimetral se debe situar a una profundidad de al menos 0,5 metros y estar separado de la fachada del edificio como mínimo I metro. Desde este anillo se deben prever las conexiones necesarias para los bajantes y para las barras de equipotencialización (Casas, 2005).

Generalmente, la configuración de tipo B se recomienda para sistemas de captación enmallados (ver IEC 62305-4, 20I0, Anexo C), estructuras con peligro de incendio y explosión (ver IEC 62305-3, 20 I0, capítulo 5.4.2), terrenos de roca sólida al descubierto, estructuras que usan material aislante en ladrillo o madera sin cimentación de acero reforzado. Esta configuración también realiza la función de igualar el potencial entre los bajantes al del nivel de tierra durante una descarga a tierra del rayo. Debido a que durante este evento se presenta una distribución desigual de las corrientes del rayo por los bajantes no equipontencializados, presentando así los diferentes potenciales, este caso ocurre a menudo en sistemas con múltiples puntas de captación y bajantes, pues cada camino por el que se drena la corriente mostrará un valor de impedancia diferente y de esta manera una caída de potencial diferente. Es por ello que la IEC 62305-3, 2010 recomienda que en estructuras provistas de aparatos eléctricos no sensibles se utilice una disposición de puesta a 


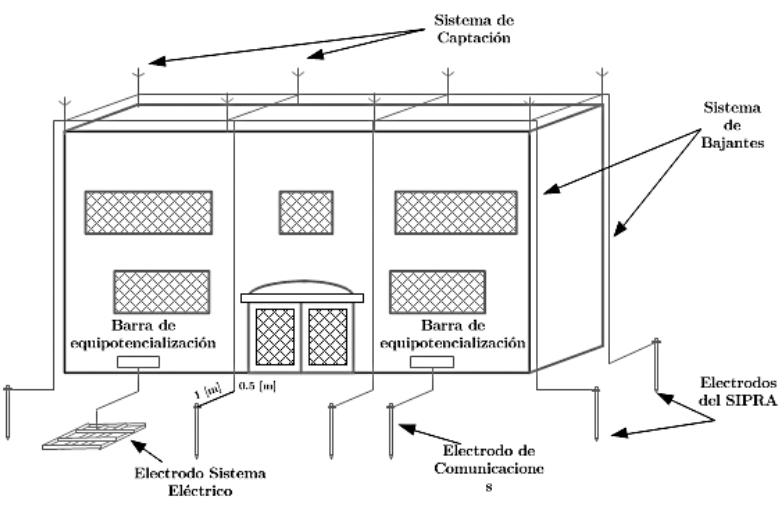

Figura 8. Esquema general de la configuración tipo A.

Fuente: Erico, 2008

tierra tipo A, pero en sistemas de equipos sensibles o sistemas electrónicos recomienda una disposición de tierra tipo B (como el caso de un centro de comunicaciones o procesamiento de datos), pues esta última provee una mayor equipotencialización de la instalación debido a su construcción. La figura 9 muestra un esquema general de la configuración tipo B en una edificación.

\section{Barra de equipotencialización (Bonding Bar)}

La barra de equipotencialización (figura I0), en una red de equipotencialización (figura II), es un elemento de material conductor en el cual se agrupan partes metálicas no energizadas de una instalación o parte de ella. En cada instalación debe existir una barra de equipotencialización principal, la cual debe estar conectada directamente a un electrodo del sistema de puesta a tierra. Para instalaciones con abundante equipamiento sensible, como en el caso de sistemas de comunicaciones, se recomienda tener una barra de equipotencialización para los equipos eléctricos en general y otra exclusiva para los equipos de comunicaciones (IEC 62305-4, 20I0).

De igual manera, estas barras generalmente se instalan para la interconexión de todos los servicios conductivos que entran en una ZPR ${ }^{14}$ (directamente o mediante el uso adecuado de DPS), junto con el conductor de protección a tierra (PE), los componentes metálicos del sistema interno (por

I4 Zona de protección contra rayos. Lightning Protection Zone, en idioma inglés.

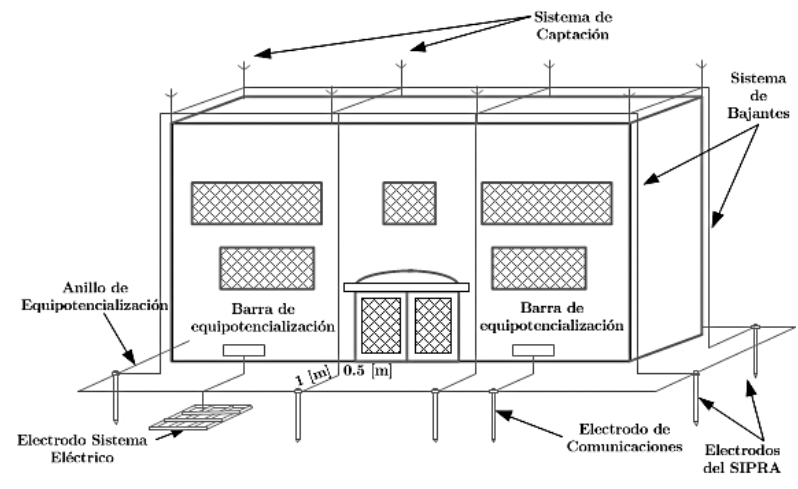

Figura 9. Esquema general de la configuración tipo B.

ejemplo, gabinetes, recintos, estantes) y el blindaje magnético de las ZPR ubicado en la periferia y dentro de la estructura.

A continuación, se citan las reglas básicas de instalación de las barras de equipotencialización (BE) para una conexión eficiente, teniendo en cuenta una baja impedancia en la red de equipotencialización:

I. La base para todas las medidas de equipotencialización es una baja impedancia en la red de equipotencialización.

2. Las barras de equipotencialización deben ser conectadas al sistema de puesta a tierra por el camino más corto posible.

3. El material y las dimensiones de la barra de equipotencialización y de los conductores de

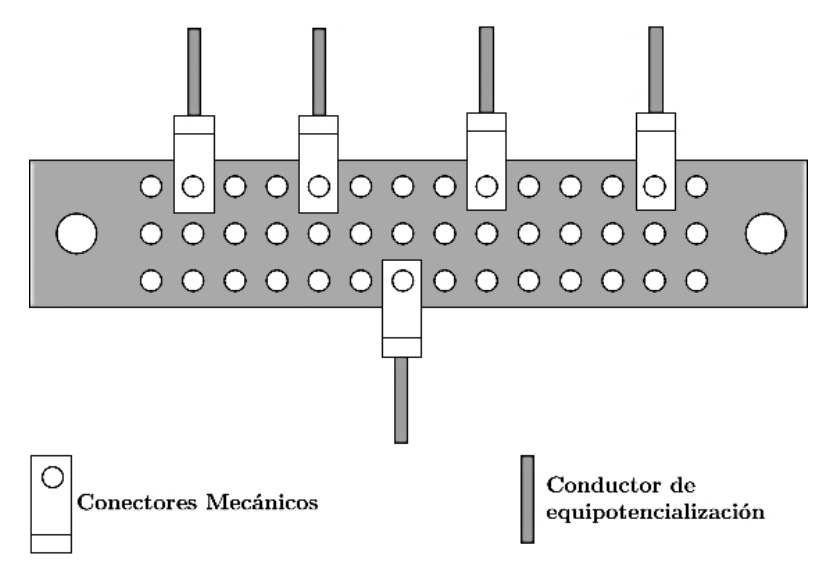

Punto de conexión

Figura 10. Barra de equipotencialización. Fuente: Erico, 2008 


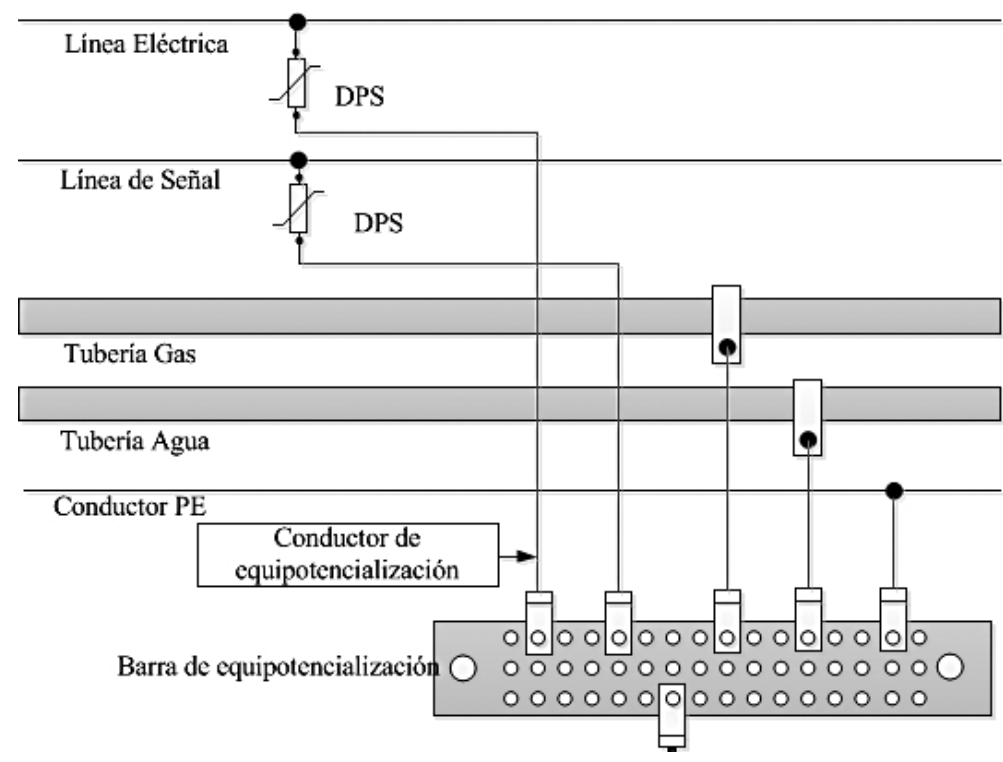

Figura II. Arreglo de red de equipotencialización. Fuente: Erico, 2008.

interconexión deben cumplir con la Tabla 2 Materiales y dimensiones para los componentes de equipotencialización presentada en la IEC 62305-4, 2010.

4. Los DPS deben ser instalados de tal manera que se use la conexión más corta posible a la barra de equipotencialización, así como a conductores vivos. Esto minimiza los cambios de tensión inductivos.

5. Sobre el lado protegido del circuito (aguas abajo de un DPS), deben ser minimizados los efectos de inducción mutua, ya sea minimizando el área del bucle o utilizando cables o ductos portacables blindados, dado que la sobretensión generada al incidir un campo magnético sobre la instalación es directamente proporcional al área de los bucles producidos por el enrutamiento de las líneas.

\section{Conclusiones}

Un diseño en el que se toman medidas de protección, orientadas principalmente a la reducción del riesgo de daño físico en estructuras y la seguridad de los seres vivos, pero en el que se desprecian de cierta forma los problemas inherentes a la calidad de la energía y la incompatibilidad electromagnética de sistemas eléctricos y electrónicos, puede no ser eficiente cuando la instalación está conformada por cargas sensibles, trayendo consigo gastos adicionales en las etapas siguientes de operación y mantenimiento.

El diseño completo de un sistema de puesta a tierra que esté compuesto por una red de equipotencialización o bonding network y un sistema de terminales de puesta a tierra puede ser la primera opción para la mitigación de los fenómenos causados por el impulso electromagnético del rayo, como son las sobretensiones de tipo conducido, eliminando las tensiones de paso y contacto peligrosas para las personas y el equipo dentro de la instalación.

Puesto que para un diseño según el enfoque de la temática del earthing and bonding es necesario utilizar el acero de refuerzo de la estructura y demás componentes metálicos de ésta, durante el diseño del sistema de puesta a tierra se deben vincular diseñadores de la estructura, ingenieros y arquitectos encargados de las obras civiles.

\section{Agradecimientos}

Los autores agradecen a la Universidad Industrial de Santander, por su apoyo al presente trabajo de investigación a través del proyecto DIEF VIE-5566: Propuesta para el diseño de instalaciones eléctricas de uso final mediante la incorporación de criterios legales, reglamentarios, normativos y técnicos considerando parámetros CEM. 


\section{Referencias}

Baggini, A.. \& Bua, F. (2004). Costes Análisis de las inversiones en soluciones para la calidad de la energía. Disponible en: http://www.leonardo-energy.org/espanol/lee-guia_calidad/ Guia\%20Calidad\%202-5\%20Costes\%20-\%20Analisis\%20 Inversiones.pdf.

Casas, F. (2005). Tierras: Soporte de la seguridad eléctrica. Bogotá: Editorial ICONTEC.

Chapman, D. (200 I). El coste de una mala calidad de la energía. Disponible en:http://www.leonardo-energy.org/espanol/leeguia_calidad/Guia\%20Calidad\%202-1\%20Costes.pdf.

Erico. (2008). Protección eléctrica de instalaciones. Disponible en: http://www.erico.com/public/library/fep/LT I 05 I.pdf.

Escalera, G. (2006). La responsabilidad social corporativa y la compatibilidad electromagnética en un contexto internacional. Madrid. Disponible en: http://redalyc.uaemex.mx/redalyc/ pdf/| 954/| 954| 5 | 85005.pdf.
IEC TR 6I000-5-6. (2002). Electromagnetic compatibility (EMC) -Part 5-6: Installation and mitigation guidelines - Mitigation of external EM influences. . International Electrotechnical Commision.

IEC 62305-3. (2010). Protection against lightning - Part 3: Physical damage to structures and life hazard. International Electrotechnical Commission.

IEC 62305-4. (2010). Protection against lightning - Part 4: Electrical and electronic systems within structures. International Electrotechnical Commission.

Langguth, W. (2004). Basic Principles of Physics for Electromagnetic Compatibility (EMC) and their Application to Electrical Installations in Buildings. University of Applied Sciences.

Schneider Electric. (2004). Puesta a tierra y compatibilidad electromagnética de los sistemas de automatización - Fundamentos y medidas - Manual de usuario. 